\title{
Reconstruction of Partitions
}

\author{
Oliver Pretzel \\ Department of Mathematics, Imperial College, \\ London, SW7 2AZ, United Kingdom, \\ and \\ Johannes Siemons*
}

School of Mathematics, University of East Anglia, Norwich, NR4 7TJ, United Kingdom

Submitted: Mar 22, 2005; Accepted: Sep 19, 2005; Published: Sep 28, 2005 Mathematics Subject Classification: 05A17, 05C60

\begin{abstract}
For the partition $x=\left[x_{1} \geq x_{2} \geq \cdots \geq x_{k}\right]$ of the integer $n=\sum_{i} x_{i}$ a $t$-deletion is a partition $y=\left[y_{1} \geq y_{2} \geq \cdots \geq y_{k}\right]$ with $x_{i} \geq y_{i} \geq 0$ and $\sum_{i}\left(x_{i}-y_{i}\right)=t$. We prove that all partitions of $n$ are reconstructible from their $t$-deletions if $n$ is sufficiently large in relation to $t$.
\end{abstract}

KEYWORDs: Partitions, reconstruction.

\section{Introduction and Notation}

The question that we address in this note is when a partition of an integer is reconstructible from certain of its subpartitions. Such reconstruction problems arise naturally, for instance in the representation theory of symmetric and Lie groups where partitions index the irreducible representations of such groups. This will be explained in the next section.

The theorem we intend to prove was announced in [3], to which we refer the reader for a detailed discussion of partition reconstruction and further results. It states that for all $t$ and all $n$ sufficiently large in relation to $t$, any partition of $n$ is fully determined by those of its subpartitions whose sum is $n-t$. In contrast to some other reconstruction problems we do not consider the multiplicity with which subpartitions occur.

In order to formulate the result precisely we make a few definitions.

\footnotetext{
${ }^{*}$ Research carried out as part of the project Reconstruction Indices of Permutation Groups funded by the Leverhulme foundation.
} 
Definitions. A partition of the integer $n$ is a sequence $x=\left[x_{1} \geq x_{2} \geq \cdots \geq x_{k}\right]$ of integers $x_{i} \geq 0$ with $|x|:=\sum_{i=1}^{k} x_{i}=n$. To avoid cumbersome distinctions we identify two partitions if they differ only in the number of zero terms. If $y=\left[y_{1} \geq y_{2} \geq \ldots \geq y_{\ell}\right]$ is a partition, then $y$ is a subpartition of $x$, denoted $y \leq x$, if $y_{i} \leq x_{i}$ for all $i=1, \ldots, \max \{k, \ell\}$. We also say $x$ contains $y$. A subpartition $y \leq x$ with $|y|=|x|-|t|$ is called a $t$-deletion of $x$.

We represent partitions by their Ferrers diagrams in the usual way. Thus the diagram of $x=\left[x_{1}, x_{2}, \ldots, x_{k}\right]$ is the set of nodes in the plane given by $F(x):=\left\{\left(j_{i},-i\right): 1 \leq\right.$ $\left.i \leq k, 1 \leq j_{i} \leq x_{i} ; i, j \in \mathbb{N}\right\}$. In particular, $y \leq x$ exactly when $F(y) \subseteq F(x)$. The meet of the partitions $x$ and $z$ is the partition $x \wedge z$ with $F(x \wedge z)=F(x) \cap F(z)$, in other words $(x \wedge z)_{i}=\min \left\{x_{i}, z_{i}\right\}$. We say that $x$ is reconstructible from its $t$-deletions if $\{y \leq x:|y|=|x|-t\}=\left\{y \leq x^{\prime}:|y|=\left|x^{\prime}\right|-t\right\}$ and $\left|x^{\prime}\right|=|x|$ imply $x^{\prime}=x$.

\section{The Theorem}

We can now state our theorem precisely.

THEOREM. Let $t>0$ be given and suppose that $n \geq 2(t+3)(t+1)$. Then every partition of $n$ is reconstructible from its $t$-deletions.

It would be nice to know if this bound is best possible in general. The result in [3] shows that the partition $x$ is reconstructible if $x$ has sufficiently many distinct $t$-deletions. That is, if $|\{y \leq x:|y|=|x|-t\}|$ is sufficiently large in terms of $n$ and $t$, see the details there and Levenshtein's paper [2].

Before we prove the theorem we discuss an application to the representation theory of symmetric groups and other Lie-type groups. What emerges is a recognition problem for the irreducible representations of a finite group which may be of independent interest.

\section{A Reconstruction Problem for Characters}

Let $G$ be a finite group and let $\chi$ be a character of $G$ over an arbitrary field. It is a standard technique of representation theory to study the restriction of a character to various subgroups of $G$. So, if $H$ is a subgroup of $G$ let $\chi_{\downarrow_{H}}$ denote the restriction of $\chi$ to $H$. Let also $\operatorname{Irr}\left(\chi_{\downarrow_{H}}\right)$ denote the set of all irreducible characters of $H$ appearing in $\chi_{\downarrow_{H}}$ with multiplicity $>0$.

It is natural to regard two characters of $G$ as equivalent, $\chi \sim_{H} \psi$, if and only if $\operatorname{Irr}\left(\chi_{\downarrow_{H}}\right)=\operatorname{Irr}\left(\psi_{\downarrow_{H}}\right)$. We may fix a suitable class $\mathcal{H}$ of subgroups of $G$ and write $\chi \sim_{\mathcal{H}} \psi$ if $\chi \sim_{H} \psi$ for all $H \in \mathcal{H}$. This relation leads to the following reconstruction problem: If $\chi$ and $\psi$ are irreducible characters of $G$, when does $\chi \sim_{\mathcal{H}} \psi$ imply $\chi=\psi$ ? In other words, when are the restrictions to subgroups in $\mathcal{H}$ sufficient to distinguish two characters? 
Let now $G$ be the symmetric group of degree $n$ and view $H \subseteq G$ as a permutation group on $\{1, \ldots, n\}$. It is well-known that many permutational properties of $H$ can be described in terms of the restriction of characters of $G$. (This applies for instance to multiple homogeneity and multiple transitivity of $H$, and so on.)

In this situation, when $G=\mathrm{Sym}_{n}$, our theorem solves the character recognition problem for the case when $\mathcal{H}$ consists of a single group $H$, this being the stabilizer of some given $t \geq 1$ points. If $\chi=\chi_{x}$ denotes the irreducible character of $G$ which is afforded by the partition $x$ of $n$ then the decomposition of $\chi_{\downarrow_{H}}$ into irreducibles is given by a branching rule, see for instance Theorem 9.2 in [1]. From this branching rule we obtain $\operatorname{Irr}\left(\chi_{\downarrow_{H}}\right)=\left\{\chi_{y}: y<x,|y|=|x|-t\right\}$ and so it follows that $\chi_{x}$ is identified uniquely by its restriction to $H$ if and only if $x$ is reconstructible from its $t$-deletions.

Similar observations can be made for families $\left\{G_{n}\right\}_{n \in \mathbb{N}}$ of Lie-type groups when the irreducible linear representations can be indexed by partitions of $n$. For instance, when $G_{n}=G L(n, q)$ some details can be found in Chapter 26 of [1]. More generally, whenever the branching rule for $\left\{G_{n}\right\}_{n \in \mathbb{N}}$ is given in terms of partition deletion, then the recognition problem for irreducible characters corresponds to a partition reconstruction problem.

\section{The Proof}

The key to our proof is a construction introduced in [3].

DEFINITION. For each positive integer $m$ we define the universal partition

$$
S(m):=\left[m,\left\lfloor\frac{m}{2}\right\rfloor,\left\lfloor\frac{m}{3}\right\rfloor, \ldots,\left\lfloor\frac{m}{m-2}\right\rfloor,\left\lfloor\frac{m}{m-1}\right\rfloor, 1\right]
$$

of the integer $s(m)=\sum_{i=1}^{m}\left\lfloor\frac{m}{i}\right\rfloor$ (where $\left\lfloor\frac{a}{b}\right\rfloor$ is the integer part of $\frac{a}{b}$ ). It is easy to see that $S(m)$ is the unique smallest partition containing all partitions of $m$. From this the following lemma follows immediately.

LEMMA 1. Let $0 \leq t \leq n$ be integers and let $x$ be a partition of $n$. Then the meet $x \wedge S(n-t)$ is the smallest partition containing all the $t$-deletions of $x$.

REMARK. In [3] the converse of the lemma [3, Lemma 2.1] is proved and used to establish the existence of partitions that are not reconstructible from their $t$-deletions.

We shall prove our theorem in a sequence of lemmas. For notational convenience we change notation letting $m=n-t$ and show that if $m+t \geq 2(t+3)(t+1)$ then every partition $x$ with $|x|=m+t$ is reconstructible from its $t$-deletions. By Lemma 1 this is the case if and only if $u:=x \wedge S(m)$ determines $x$. Therefore, in terms of Ferrers diagrams, we need to show that there is at most one way to adjoin $t_{0}:=(m+t)-|u|$ nodes to $u$ to obtain a partition $y$ of $m+t$ with $u=y \wedge S(n)$. We therefore make the following ad hoc definition. 
Definition. Let $u$ be a subpartition of $S(m)$. We call $y$ an extension (or more precisely, $t$-extension) of $u$, if $|y|=m+t$ and $y \wedge S(m)=u$.

So what we have to show is that if $m+t \geq 2(t+3)(t+1)$ then any $u \leq S(m)$ has at most one extension.

From now on partitions are identified with their Ferrers diagrams. First we shall characterize the initial nodes that can be adjoined to $u$ in forming an extension and call them corners. A corner must be a node that can be adjoined to $u$ producing a diagram representing a partition $y^{\prime}$, and it must lie outside $S(m)$ so that the intersection $y^{\prime} \wedge S(n)$ is still $u$. We phrase these conditions more formally in two further ad hoc definitions as follows.

Definitions. Let $u$ be a subpartition of $S(m)$. The node $(k, \ell)$ (in row $k$ and column $\ell$ of the $n \times n$ grid) is a corner of $u$ if

(a) $\ell \leq u_{k^{\prime}}$ for all $1 \leq k^{\prime}<k$, and

(b) $\quad \ell=u_{k}+1=(S(m))_{k}+1$.

Thus corners are the top left hand corner nodes of the difference diagrams $y \backslash S(m)=$ $y \backslash u$ where $y$ runs through the extensions of $u$.

If $(k, \ell)$ is a corner, let $b(k, \ell)$ be the block of all nodes $\left(k^{\prime}, \ell^{\prime}\right)$ with $k^{\prime} \leq k, \ell^{\prime} \leq \ell$ except $(k, \ell)$ itself. In particular, $b(k, \ell) \leq u$.

The proof of our theorem now follows from a sequence of lemmas. The first lemma shows in passing that small subpartitions of $S(m)$ have no corners at all.

Lemma 2. Let $(k, \ell)$ be a corner of $u \in S(m)$. Then the block $b(k, \ell)$ contains at least $m-1$ nodes.

Proof. The number of nodes is $k \cdot\left(\left\lfloor\frac{m}{k}\right\rfloor+1\right)-1 \geq k \cdot \frac{m}{k}-1=m-1$.

If $|u|=m+t$, then $u=x$ is the only extension of $u$, and if $|u|>m+t$ then $u$ has no extension. In either case there is nothing to prove. We therefore assume from now on that $|u|<m+t$. Next we complete the proof when $u$ has exactly one corner. For this part of the proof we do not need the full bound on $m+t$, but only the weaker condition $m>t^{2}$.

Lemma 3. Let $u \leq S(m)$ have exactly one corner $c=(k, \ell)$ and let $|u|<m+t$ and $m>t^{2}$. Then $u$ has exactly one extension $x$.

Proof. By Lemma 2, $b(k, \ell) \cup\{c\}$ accounts for at least $m$ nodes of any extension $y$. As $c$ is the only corner of $u$ all further nodes to be added must lie to the right of $c$ or below it (or both). Let $c^{\prime}$ be the next node to be adjoined, thus $c^{\prime}=(k, \ell+1)$ or $c^{\prime}=(k+1, \ell)$. 
If $k>t$ then $c^{\prime}=(k, \ell+1)$ is impossible; for in this case all nodes in the column above $c^{\prime}$ must belong to $u$. So $|y| \geq|b(k, \ell)|+1+k>m+t$. Thus when $k>t$ all additional nodes are in the same column as $c$ and below it.

Similarly, when $k \leq t$ then $c^{\prime}=(k+1, \ell)$ is impossible for the following reason. By hypothesis $m>t^{2}$ and hence $m / k>t$. Arguing as before, we see that if $y$ contains $c^{\prime}$ then $|y| \geq|b(k, \ell)|+1+\ell=m+\left\lfloor\frac{m}{k}\right\rfloor+1>m+t$. Thus for $k \leq t$ all additional nodes of an extension must be in the same row and to the right of $c$.

In either case $u$ can be extended in only one way and, since the number of nodes to be added is fixed, that concludes the proof.

It remains to show that for sufficiently large $m$, subpartitions $u \leq S(m)$ can have no more than one corner.

LEMma 4. Let $u \leq S(m)$ and $|u|<m+t$. If $m \geq(t+2)(2 t+3)$, then $u$ has at most one corner.

Proof. We shall assume that $u$ has two corners and derive a contradiction. Thus suppose that $c=(k, \ell)$ and $c^{\prime}=\left(k^{\prime}, \ell^{\prime}\right)$ with $k<k^{\prime}$ and $\ell=\left\lfloor\frac{m}{k}\right\rfloor+1>\ell^{\prime}=\left\lfloor\frac{m}{k^{\prime}}\right\rfloor+1$ are corners. Then $b(k, \ell) \cup b\left(k^{\prime}, \ell^{\prime}\right) \leq u$. Let $d=b(k, \ell) \backslash b\left(k^{\prime}, \ell^{\prime}\right)$ be the set of nodes in $b(k, \ell)$ but not in $b\left(k^{\prime}, \ell^{\prime}\right)$. Then $|d|=k \cdot\left(\ell-\ell^{\prime}\right)-1$ For a contradiction it suffices to show that $d>t$, for then $|u| \geq\left|b\left(k^{\prime}, \ell^{\prime}\right)\right|+d \geq m+t$.

We shall show $d>t$ using two different estimates, depending on the size of $k$. The first is the trivial observation that $|d| \geq k-1$ (since $b(k, \ell)$ contains the nodes above $c$, which are not in $\left.b\left(k^{\prime}, \ell^{\prime}\right)\right)$. Hence $|d|>t$ for $k>t+1$.

The second estimate assumes that $k \leq t+1$ and uses the values for $\ell$ and $\ell^{\prime}$ calculated above.

$$
\begin{aligned}
d & =k \cdot\left(\ell-\ell^{\prime}\right)-1 \\
& =k \cdot\left(\left\lfloor\frac{m}{k}\right\rfloor-\left\lfloor\frac{m}{k^{\prime}}\right\rfloor\right)-1 \\
& \geq k \cdot\left(\left\lfloor\frac{m}{k}\right\rfloor-\left\lfloor\frac{m}{k+1}\right\rfloor\right)-1 \\
& \geq k \cdot\left(\frac{m}{k}-\frac{m}{k+1}-1\right)-1 \\
& =k \cdot\left(\frac{m}{k(k+1)}-1\right)-1 \\
& =\frac{m}{k+1}-(k+1) \\
& \geq \frac{m}{t+2}-(t+2),
\end{aligned}
$$

In order that in this case $|d|>t$ it is sufficient that $m /(t+2)>(2 t+2)$ or equivalently $m \geq(t+2)(2 t+3)$. 
Proof of theorem. We have shown that for $m \geq(t+2)(2 t+3)$ any subpartition $u$ of $S(m)$ has at most one corner (Lemma 4 ) and, since then automatically $m>t^{2}$, $u$ has at most one extension (Lemma 3). The proof of the theorem thus reduces to the simple observation that $m \geq(t+2)(2 t+3)$ is equivalent to $m+t \geq(t+2)(2 t+3)+t=2(t+3)(t+1)$.

Hence for partitions $x$ with $|x|=n:=m+t \geq 2(t+3)(t+1)$, the meet $u=x \wedge S(m)$ determines $x$ and our reconstruction theorem is proved.

\section{References}

[1] James, G., The Representation Theory of the Symmetric Groups, Lecture Notes in Mathematics, Springer Verlag, Berlin Heidelberg New York, 1978.

[2] Levenshtein, V., Efficient reconstruction of sequences from their subsequences and supersequences, J. Combin. Theory Ser. A 93 (2001) 310-332.

[3] Maynard,P. and Siemons J., Efficient reconstruction of partitions, Discr. Math. 293 (2005) 205-211. 